\title{
Influência da freqüência alimentar no desempenho de juvenis de jundiá
}

\author{
Régis Canton ${ }^{1}$, Marcos Weingartner ${ }^{1}$, Débora Machado Fracalossi ${ }^{1}$, Evoy Zaniboni Filho ${ }^{1}$ \\ ${ }^{1}$ Laboratório de Biologia e Cultivo de Peixes de Água Doce, Departamento de Aqüicultura, Universidade Federal de Santa Catarina. Rodovia \\ Admar Gonzaga, 1346. Bairro Itacorubi, CEP: 88034-001, Florianópolis, SC. Fone-Fax: 48-3389-5216.
}

RESUMO - Com objetivo de avaliar a influência da freqüência alimentar no ganho de peso, na taxa de crescimento específico, na conversão alimentar e na sobrevivência de juvenis de jundiá, 12 grupos de 280 peixes $(7,47 \pm 1,73$ g) foram estocados em viveiros de terra $\left(2\right.$ peixes $\left./ \mathrm{m}^{2}\right)$. Uma dieta comercial extrusada, com $42 \%$ de $\mathrm{PB}$, foi fornecida até a aparente saciedade em quatro regimes de arraçoamento: 1, 2, 3 e 4 vezes ao dia, em triplicata, durante 120 dias (maio a setembro de 2003). O ganho de peso médio foi determinado por meio de biometrias (10\% da população) realizadas em intervalos de aproximadamente 40 dias. A qualidade da água foi monitorada durante o período experimental para obtenção das médias de temperatura, oxigênio dissolvido, $\mathrm{pH}$, transparência, amônia, alcalinidade e dureza: $18,1 \pm 0,1^{\circ} \mathrm{C}, 7,9 \pm 0,2 \mathrm{mg} / \mathrm{L}, 8,0 \pm 0,8$, $109 \pm 29 \mathrm{~cm}, 0,5 \mathrm{mg} / \mathrm{L}, 46 \mathrm{mg} / \mathrm{L} \mathrm{CaCO}_{3}$ e $40 \mathrm{mg} / \mathrm{L} \mathrm{CaCO}_{3}$, respectivamente. O ganho de peso e a taxa de crescimento específico dos juvenis aumentaram com o aumento da freqüência alimentar. O ganho de peso e a taxa de crescimento específico diferiram entre os juvenis alimentados 1 e 4 vezes/dia. Os valores de conversão alimentar não diferiram significativamente entre as freqüências alimentares, porém, a sobrevivência foi significativamente maior no grupo de peixes alimentados 3 vezes/dia, em comparação àqueles alimentados 1 vez/dia. Recomenda-se a alimentação de juvenis de jundiá (8 a 45 g) pelo menos 2 vezes/dia quando cultivados em temperatura média da água de $18^{\circ} \mathrm{C}$.

Palavras-chave: alimentação, bagre, crescimento, piscicultura, viveiro

\section{Effect of feeding frequency on performance of jundiá juveniles}

\begin{abstract}
The influence of feeding frequency was evaluated on weight gain, specific growth rate, feed conversion and survival of jundiá juveniles. Twelve groups of 280 fish $(7.47 \pm 1.73 \mathrm{~g})$ were each stocked in twelve ponds $\left(2 \mathrm{fish} / \mathrm{m}^{2}\right)$ and fed to apparent satiety an extruded commercial feed containing 42\% CP In four feeding schedules: 1, 2, 3 and 4 times a day, in triplicate, for 120 days (May to September, 2003). Average weight gain was recorded by sampling $10 \%$ of the total fish/ pond approximately every forty days. Water quality was monitored throughout the experimental period by measuring temperature, dissolved oxygen, $\mathrm{pH}$, turbidity, ammonia, alkalinity and hardness which were $18.1 \pm 0.1^{\circ} \mathrm{C}, 7.9 \pm 0.2 \mathrm{mg} / \mathrm{L}$, $8.0 \pm 0.8,109 \pm 29 \mathrm{~cm}, 0.5 \mathrm{mg} / \mathrm{L}, 46 \mathrm{mg} / \mathrm{L} \mathrm{CaCO}_{3}$, and $40 \mathrm{mg} / \mathrm{L} \mathrm{CaCO}_{3}$, respectively. Fish weight gain and specific growth rate increased as feeding frequency increased. Juveniles fed four times a day showed significantly higher weight gain and specific growth rate than fish fed just once a day. Feed conversion did not differ significantly among feeding frequencies, however survival was significantly higher for the groups fed three times a day when compared to the ones fed only once a day. Therefore, jundiá juveniles weighing from 8 to 45 g should be fed at least twice a day, when raised in earthen ponds at an average water temperature of $18^{\circ} \mathrm{C}$.
\end{abstract}

Key Words: catfish, feeding, fish culture, growth, pond

\section{Introdução}

O jundiá (Rhamdia quelen) é um bagre de água doce, onívoro e de ampla distribuição geográfica, ocorrente desde a região central da Argentina até o sul do México. Silfvergrip (1996) relatou que o gênero Rhamdia é constituído de 11 espécies e que, para a espécie $R$. quelen, são descritas 49 sinonímias. Esta espécie tem despertado grande interesse para a piscicultura da região Sul do Brasil por seu crescimento acelerado, inclusive nos meses mais frios (Carneiro et al., 2002; Fracalossi et al., 2002, 2004), sua excelente eficiência alimentar (Meyer \& Fracalossi, 2004), sua resistência ao manejo, além de sua carne saborosa, sem espinhos intramusculares. Verifica-se grande oferta desta espécie na região Sul, onde tem sido cultivada com razoável sucesso, porém, são poucos os estudos sobre seu manejo alimentar e suas exigências nutricionais.

A influência da freqüência alimentar sobre o desenvolvimento de juvenis tem sido estudada em várias espécies (Tsevis et al., 1992; Wang et al., 1998; Lee et al., 2000a; Lee 
et al., 2000b; Dwyer et al., 2002), sendo normalmente observado aumento no ganho de peso quando juvenis são alimentados mais de uma vez ao dia. Entretanto, Carneiro \& Mikos (2005) não detectaram diferença significativa no ganho de peso, na taxa de crescimento específico e na conversão alimentar entre grupos de juvenis de jundiá com peso médio inicial de 1,88 g quando alimentados 1,2 , 3 ou 4 vezes ao dia, durante 65 dias, em viveiros com temperatura média de água de $25,2 \pm 1,3^{\circ} \mathrm{C}$. Desse modo, objetivou-se investigar o efeito de diferentes freqüências alimentares sobre o desempenho de juvenis de jundiá (peso médio inicial 7,47 g) alimentados em viveiros de terra durante 120 dias.

\section{Material e Métodos}

O experimento foi realizado na Estação de Piscicultura de São Carlos, região oeste do estado de Santa Catarina, vinculada ao Laboratório de Biologia e Cultivo de Peixes de Água Doce, Universidade Federal de Santa Catarina, no período de maio a setembro de 2003.

Grupos de 280 juvenis de jundiá com peso inicial de $7,47 \pm 1,73$ g, obtidos de uma piscicultura comercial, foram estocados em 12 viveiros e aclimatados às condições experimentais durante 15 dias. Os viveiros possuíam parede de alvenaria e fundo de terra, área de $140 \mathrm{~m}^{2}$, com abastecimento e saída de água individual. Os peixes ( $10 \%$ do total de peixes de cada viveiro) foram pesados em intervalos de 40 dias para estimativa do ganho de peso, após jejum de 24 horas. Ao final de 120 dias, uma amostragem semelhante foi realizada para obtenção do peso individual, enquanto o restante da população de cada viveiro foi pesado em grupo para determinação do ganho de peso total no período.

Durante o período de aclimatação, os juvenis foram alimentados com uma dieta comercial extrusada contendo 45\% de PB e 2 mm de diâmetro. Posteriormente, durante o período experimental, os peixes receberam dieta comercial extrusada (4,2 mm de diâmetro) contendo 42\% PB, até aparente saciedade, em quatro regimes de arraçoamento: 1 , 2, 3 ou 4 vezes ao dia, em triplicata (Tabela 1).

Os horários de alimentação foram estabelecidos para os peixes alimentados 1 , 2 e 3 vezes ao dia, considerando o período do dia em que a temperatura era mais alta. O peso do alimento oferecido durante o ensaio alimentar foi registrado diariamente para cada viveiro para posterior cálculo da conversão alimentar.

A partir dos registros do consumo total de ração e do ganho de peso [GP = (peso final - peso inicial)], foram calculadas a taxa de crescimento específico [TCE $=100 \times(\ln$
Tabela 1 - Horários das alimentações diárias

Table 1 - Feeding schedule for each feeding frequency tested

\begin{tabular}{lcccc}
\hline $\begin{array}{l}\text { Freqüência alimentar diária } \\
\text { Daily feeding frequency }\end{array}$ & \multicolumn{4}{c}{$\begin{array}{c}\text { Horário de alimentação } \\
\text { Feeding schedule }\end{array}$} \\
\hline 1 & - & - & - & $17 \mathrm{~h}$ \\
2 & - & $11 \mathrm{~h}$ & - & $17 \mathrm{~h}$ \\
3 & - & $11 \mathrm{~h}$ & $14 \mathrm{~h}$ & $17 \mathrm{~h}$ \\
4 & $8 \mathrm{~h}$ & $11 \mathrm{~h}$ & $14 \mathrm{~h}$ & $17 \mathrm{~h}$
\end{tabular}

peso médio final - ln peso médio inicial)/tempo (dia)] e a conversão alimentar [CA = (consumo de ração/ganho de peso)]

Durante todo o período experimental, a temperatura e a concentração de oxigênio dissolvido na água foram medidas diariamente (8 e 17h). Semanalmente, foram monitorados a transparência e o $\mathrm{pH}$ e, mensalmente, a alcalinidade, a dureza e a presença de amônia. As médias foram 7,9 \pm 0,2 mg/L para oxigênio, 8,0 \pm 0,8 para $\mathrm{pH}$, $109 \pm 29 \mathrm{~cm}$ para transparência, $46 \mathrm{mg} / \mathrm{L} \mathrm{CaCO}_{3}$ para alcalinidade, $40 \mathrm{mg} / \mathrm{L} \mathrm{CaCO}_{3}$ para dureza e $0,5 \mathrm{mg} / \mathrm{L}$ para amônia total. A temperatura média da água foi de $18,1 \pm 0,1^{\circ} \mathrm{C}$ e indica que não houve limitação para o desenvolvimento dos juvenis de jundiá (Baldisserotto \& Radünz Neto, 2004).

Os dados de ganho de peso, taxa de crescimento específico, conversão alimentar e sobrevivência foram submetidos à análise de variância. Quando houve diferença significativa entre os tratamentos, as médias foram comparadas pelo teste Tukey a 5\% de significância.

\section{Resultados e Discussão}

Os resultados de ganho de peso e conversão alimentar obtidos neste estudo comprovam o grande potencial desta espécie em comparação a outras de peixes cultivados. O ganho de peso médio dos juvenis foi de $21,17 \pm 1,98 \mathrm{~g}$; $28,35 \pm 0,06 \mathrm{~g} ; 31,86 \pm 6,67 \mathrm{~g} \mathrm{e} 41,10 \pm 4,56 \mathrm{~g}$ para os grupos alimentados 1, 2, 3 e 4 vezes/dia, respectivamente (Figura 1). Com o aumento da freqüência de alimentação, houve acréscimo no ganho de peso ao longo do tempo. Portanto, ao final de 120 dias, os peixes alimentados quatro vezes ao dia ganharam praticamente o dobro do peso daqueles que receberam somente uma alimentação diária.

Carneiro \& Mikos (2005), no entanto, observaram que a freqüência alimentar (1 a 4 vezes/dia) não afetou o crescimento de juvenis de jundiá (peso médio 1,88 g) alimentados durante 65 dias em temperatura da água de $25,2 \pm 1,3^{\circ} \mathrm{C}$. A causa desta discrepância é desconhecida, pois resultados de vários estudos com juvenis de outras espécies 


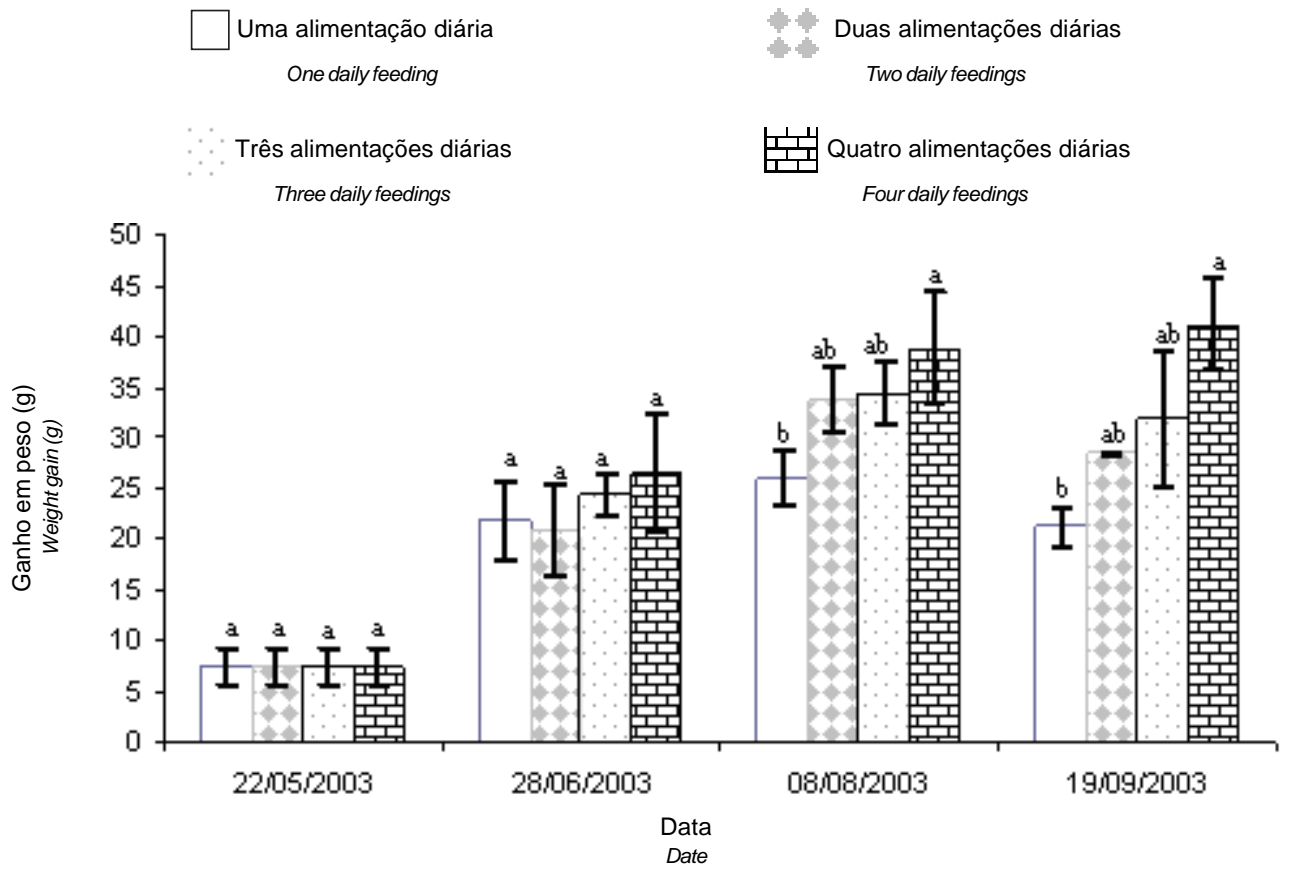

Figura 1 - Ganho de peso de juvenis de jundiá submetidos a quatro freqüências alimentares distintas. Colunas com letras diferentes em cada biometria indicam diferenças significativas $(P<0,05)$.

Figure 1 - Average weight gain of jundiá juveniles submitted to four feeding frequencies. Columns with different letters in the same date indicate significant differences $(P<0.05)$.

comprovam que o aumento da freqüência alimentar, até certo limite, proporciona aumento no ganho de peso (Andrews \& Page, 1975; Grayton \& Beamish, 1977; Tsevis et al., 1992). Neste estudo, não foi possível determinar esse limite máximo para a freqüência alimentar, pois o máximo ganho de peso ocorreu na maior freqüência de alimentação diária testada (4 vezes/dia). Resultados semelhantes associados ao aumento da freqüência de alimentação foram obtidos com híbridos de sunfish (Lepomis cyanellus fêmeas $\mathrm{x}$ L. macrochirus machos), em que juvenis com peso inicial de 3 a $8 \mathrm{~g}$ apresentaram maior ganho de peso quando alimentados 3 ou 4 vezes/dia em comparação a 1 ou 2 alimentações diárias (Wang et al., 1998). Para juvenis (6 a 8 g de peso inicial) de yellowtail flounder (Limanda ferruginea), carnívoro endêmico das águas geladas da América do Norte, o maior ganho de peso foi obtido com alimentação 2 ou 4 vezes/dia, em comparação a uma única alimentação diária ou, ainda, uma alimentação a cada dois dias (Dwyer et al., 2002). Tendência de aumento do ganho de peso foi registrada para juvenis de linguado (Paralichthys olivaceus) (peso inicial de $3,5 \mathrm{~g}$ ) com o aumento da freqüência alimentar de 1 a cada dois dias para 1, 2 ou 3 alimentações diárias durante 40 dias (Lee et al., 2000a). Entretanto, aumento significativo no ganho de peso foi observado em juvenis de Korean rockfish (Sebastes schlegeli), peso de 5 a 20 g, alimentados 1 vez/dia em comparação a duas alimentações diárias ou uma vez a cada dois dias (Lee et al., 2000b).
Andrews \& Page (1975) observaram crescimento mais rápido e maior eficiência alimentar para o bagre-do-canal (Ictalurus punctatus) alimentado 2 vezes/dia em comparação aos alimentados 24 vezes/dia. Portanto, a freqüência alimentar ótima varia com a espécie (Sampath, 1984). Estudos evidenciam que o consumo de grandes quantidades de alimento em curtos intervalos de tempo diminui a eficiência digestiva (Windell et al., 1978; Bergot \& Breque, 1983; Henken et al., 1985).

Fracalossi et al. (2004) observaram maior ganho de peso médio (106g) e taxa de crescimento específico (2,85\%) quando juvenis de jundiá (peso inicial 8,8 g) foram alimentados 2 vezes/dia (8 e 17h) em viveiros de terra na mesma região onde foi realizado este estudo (dados referentes aos meses de junho a setembro, 120 dias). Entretanto, a temperatura média da água foi $20^{\circ} \mathrm{C}$, superior à observada neste estudo $\left(18^{\circ} \mathrm{C}\right)$. Provavelmente, a infestação por íctio (Ichthyophthirius multifiliis) nos meses de agosto e setembro e as diferenças genéticas entre os lotes (fontes de juvenis distintas) contribuíram para o menor ganho de peso e a menor taxa de crescimento específico dos peixes neste estudo.

A maior taxa de crescimento específico foi apresentada pelos juvenis alimentados 4 vezes/dia (Tabela 2). Essa taxa, porém, só diferiu significativamente daquela obtida nos juvenis alimentados $1 \mathrm{vez} / \mathrm{dia}$. As taxas de crescimento específico encontradas neste estudo foram semelhantes às obtidas para o robalo europeu (Dicentrarchus labrax) 
Tabela 2 - Valores médios de taxa de crescimento específico, conversão alimentar e sobrevivência de juvenis de jundiá submetidos a quatro freqüências alimentares

Table 2 - Average specific growth rate, feed conversion and survival (meant standard deviation) of jundiá juveniles submitted to four feeding frequencies

\begin{tabular}{lcrr}
\hline $\begin{array}{l}\text { Freqüência alimentar diária } \\
\text { Daily feeding frequency }\end{array}$ & $\begin{array}{c}\text { Taxa de crescimento específico (\%) } \\
\text { Specific growth rate (\%) }\end{array}$ & $\begin{array}{c}\text { Conversão alimentar } \\
\text { Feed conversion }\end{array}$ & $\begin{array}{c}\text { Sobrevivência (\%) } \\
\text { Survival (\%) }\end{array}$ \\
\hline 1 & $1,12^{\mathrm{b}} \pm 0,06$ & $1,31 \pm 0,14$ & $70,12^{\mathrm{b}} \pm 17,21$ \\
2 & $1,31^{\mathrm{ab}} \pm 0,00$ & $1,64 \pm 0,18$ & $71,07^{\mathrm{ab}} \pm 6,81$ \\
3 & $1,38^{\mathrm{ab}} \pm 0,15$ & $1,44 \pm 0,26$ & $86,90^{\mathrm{a}} \pm 19,80$ \\
4 & $1,56^{\mathrm{a}} \pm 0,08$ & $1,54 \pm 0,20$ & $80,12^{\mathrm{ab}} \pm 17,21$ \\
\hline
\end{tabular}

ab Na mesma coluna, médias seguidas de letras diferentes indicam diferenças significativas $(P<0,05)$.

ab Means followed by different letters within columns indicate significant differences $(P<0.05)$.

(Tsevis et al., 1992) e para o bagre-australiano (Heteropneustes fossilis) (Marian et al., 1982), as quais aumentaram com o incremento da freqüência alimentar. Híbridos de sunfish alimentados 1 vez/dia também apresentaram menor taxa de crescimento específico em comparação a indivíduos alimentados 4 vezes/dia, ressaltando-se que 2 ou 3 alimentações diárias proporcionaram taxa de crescimento intermediária (Wang et al., 1998).

A conversão alimentar (Tabela 2) não diferiu significativamente entre os juvenis submetidos às diferentes freqüências alimentares. Resultados semelhantes foram registrados para híbridos de sunfish (Wang et al., 1998). Em um período similar, a conversão alimentar dos juvenis de jundiá neste estudo foi melhor que a registrada por Lovshin et al. (1990) para juvenis de tilápia (Oreochromis niloticus), de 2,4.

Neste estudo, verificou-se nos meses de agosto e setembro, redução no consumo de 44, 31, 21 e 30\% para os juvenis de jundiá alimentados 1, 2, 3 e 4 vezes/dia, respectivamente, em comparação ao consumo registrado no mês de julho. Concomitantemente à redução no consumo, observou-se perda de peso para os juvenis alimentados 1 , 2 ou 3 vezes/dia e diminuição no ganho de peso dos jundiás alimentados 4 vezes/dia (Figura 1).

As diferenças na sobrevivência (Tabela 2) entre os peixes alimentados 1, 2, 3 ou 4 vezes/dia provavelmente estão relacionadas à variação no grau de infestação do protozoário íctio (Ichthyophthirius multifiliis), em todas as unidades experimentais, com a diminuição da temperatura logo após a terceira biometria. Em uma das repetições do tratamento com duas alimentações diárias, a mortalidade chegou a $72 \%$ da população total do viveiro, e, portanto, os dados desta unidade experimental foram descartados na análise estatística. A ictiofitiríase ou doença dos "pontos brancos" é a causa das maiores perdas mundiais nas pisciculturas de água doce (Harper, 2003). O jundiá é uma espécie extremamente susceptível a esta parasitose, principalmente nas fases iniciais de desenvolvimento (Fracalossi et al., 2002) e em situações de queda na temperatura.

\section{Conclusões}

No cultivo de juvenis de jundiá (8 a 45 g) em temperatura média da água de $18^{\circ} \mathrm{C}$, recomenda-se freqüência de alimentação de pelo menos 2 vezes/dia.

\section{Agradecimento}

À direção e aos funcionários da Estação de Piscicultura de São Carlos (município de São Carlos, SC), pelo auxílio na condução do experimento.

\section{Literatura Citada}

ANDREWS, J.W.; PAGE, J.W. The effects of frequency of feeding on culture of catfish. Transactions of the American Fisheries Society, v.104, p.317-321, 1975.

BALDISSEROTTO, B.; RADÜNZ NETO, J. Criação de jundiá. Santa Maria: Universidade Federal de Santa Maria, 2004. 232p.

BERGOT, F.; BREQUE, J. Digestibility of starch by rainbow trout: effects of physical state of starch and of the intake level. Aquaculture, v.34, p.203-212, 1983.

CARNEIRO, P.C.F.; BENDHACK, F.; MIKOS, J.D. et al. Jundiá: um grande peixe para a região Sul. Panorama da Aqüicultura v.12, p.41-46, 2002.

CARNEIRO, P.C.F.; MIKOS, J.D. Freqüência alimentar e crescimento de alevinos de jundiá, Rhamdia quelen. Ciência Rural, v.35, n.1, p.187-191, 2005.

DWYER, K.S.; BROWN, J.A.; PARRISH, C. et al. Feeding frequency affects food consumption, feeding pattern and growth of juvenile yellowtail flounder, Limanda ferruginea. Aquaculture, v.213, p.279-292, 2002.

FRACALOSSI, D.M.; MEYER, G.; SANTAMARIA, F.M. et al. Desempenho do jundiá, Rhamdia quelen, e do dourado, Salminus brasiliensis, em viveiros de terra na Região Sul do Brasil. Acta Scientiarum, v.26, n.3, p.345-352, 2004.

FRACALOSSI, D.M.; ZANIBONI FILHO, E.; MEURER, S. No rastro das espécies nativas. Panorama da Aqüicultura, v.12, p.4349, 2002.

GRAYTON, B.D.; BEAMISH, F.W.H. Effects of feeding frequency on food intake, growth and body composition of rainbow trou (Salmo gairdneri). Aquaculture, v.11, p.159-172, 1977.

HARPER, C. Introducing Ichthyophthirius multifiliis - A devastating parasite. Aquaculture Magazine, v.29, p.40-52, 2003.

HENKEN, A.M.; KLEINGELD, D.W.; TIJSSEN, P.A. T. The effect or feeding level on apparent digestibility of dietary dry mater, crude protein and gross energy in the African catfish (Claris gariepinus). Aquaculture, v.51, p.1-11, 1985. 
LEE, S.M.; CHO, S.H.; KIM, D.-J. Effects of feeding frequency and dietary energy level on growth and body composition of flounder, Paralichthys olivaceus (Temminck and Schlegel). Aquaculture Research, v.31, p.917-921, 2000a.

LEE, S.-M.; HWANG, U.G.; CHO, S.H. Effects of feeding frequency and dietary moisture content on growth and body composition and gastric evacuation of juvenile korean rockfish (Sebastes schlegeli). Aquaculture, v.187, p.399409, 2000b.

LOVSHIN, L.L.; DOUGLAS, T.; LIEUTAUD, A.O. Growth and yield of mixed-sex, young-of-the-years Oreochromis niloticus raised at two densities in earthen ponds in Alabama, USA. Aquaculture, v.89, p.21-26, 1990.

MARIAN, M.P.; PONNIAH, A.G.; PITCHAIRAJ, R. et al. Effect of feeding frequency on surfacing activity and growth in the air-breathing fish, Heteropneustes fossilis. Aquaculture, v.26, p.237-244, 1982.

MEYER, G.; FRACALOSSI, D.M. Protein requirement of jundia fingerlings, Rhamdia quelen, at two dietary energy concentrations. Aquaculture, v.240, p.331-343, 2004.

SAMPATH, K. Preliminary report on the effects of feeding frequency in Channa striatus. Aquaculture, v.40, p.301-306, 1984.
SILFVERGRIP, A.M.C. A Systematic revision of the neotropical catfih genus Rhamdia (Teleostei, Pimelodidae). Estocolmo: Department of Zoology Stockholm University, 1996. Thesis (Ph.D in Zoology) - Swedish Museum of Natural History, 1996.

TSEVIS, N.; KLAOUDATOS, S.; CONIDES, A. Food conversion budget in sea bass, Dicentrarchus labrax, fingerlings under two different feeding frequency patterns. Aquaculture, v.101, p.293-304, 1992.

WANG, N.; HAYWARD, R.S.; NOLTIE, D.B. Effect of feeding frequency on food consumption, growth, size variation, and feeding pattern of age-0 hybrid sunfish. Aquaculture, v.165, p.261-267, 1998.

WINDELL, J.T.; FOLTZ, J.W.; SAROKON, J.A. Effect of fish size, temperature, and amount fed on nutrient digestibility of a pelletet diet by rainbow trout (Salmo gairdneri). Transactions of the American Fisheries Society, v.107, p.613-616, 1978.

Recebido: 14/03/06 Aprovado: 19/12/06 